\title{
On Fully Degenerate Daehee Numbers and Polynomials of the Second Kind
}

\author{
Sang Jo Yun ${ }^{1}$ and Jin-Woo Park $\mathbb{D}^{2}$ \\ ${ }^{1}$ Department of Mathematics, Dong-A University, Busan 604-714, Republic of Korea \\ ${ }^{2}$ Department of Mathematics Education, Daegu University, Gyeongsan-si, Gyeongsangbuk-do 38453, Republic of Korea
}

Correspondence should be addressed to Jin-Woo Park; a0417001@knu.ac.kr

Received 31 May 2020; Accepted 20 July 2020; Published 2 September 2020

Academic Editor: Serkan Araci

Copyright (c) 2020 Sang Jo Yun and Jin-Woo Park. This is an open access article distributed under the Creative Commons Attribution License, which permits unrestricted use, distribution, and reproduction in any medium, provided the original work is properly cited.

In a study, Carlitz introduced the degenerate exponential function and applied that function to Bernoulli and Eulerian numbers and degenerate special functions have been studied by many researchers. In this paper, we define the fully degenerate Daehee polynomials of the second kind which are different from other degenerate Daehee polynomials and derive some new and interesting identities and properties of those polynomials.

\section{Introduction}

Let $p$ be a fixed prime number. Throughout this paper, $\mathbb{Z}_{p}$, $\mathbb{Q}_{p}$, and $\mathbb{C}_{p}$ will denote the ring of $p$-adic integers, the field of $p$-adic rational numbers, and the completion of algebraic closure of $\mathbb{Q}_{p}$, respectively.

Let $f(x)$ be a uniformly differentiable function on $\mathbb{Z}_{p}$. Then, the $p$-adic invariant integral on $\mathbb{Z}_{p}$ is defined as

$$
I_{0}(f)=\int_{\mathbb{Z}_{p}} f(x) \mathrm{d} \mu_{0}(x)=\lim _{N \longrightarrow \infty} \frac{1}{p^{N}} \sum_{n=0}^{p^{N}-1} f(x),
$$

From (1), we have

$$
\text { (see }[1-3]) \text {. }
$$

$$
\begin{aligned}
I_{0}\left(f_{n}\right)-I_{0}(f) & =\sum_{l=0}^{n-1} f^{\prime}(l), \quad \text { where } f_{n}(x) \\
& =f(x+n), f^{\prime}(l)=\left.\frac{\mathrm{d} f(x)}{\mathrm{d} x}\right|_{x=l} .
\end{aligned}
$$

In particular, if $n=1$, then

$$
I_{0}\left(f_{1}\right)-I_{0}(f)=f^{\prime}(0) .
$$

The Stirling numbers of the first kind are defined by

$$
(x)_{n}=\sum_{l=0}^{n} S_{1}(n, l) x^{l}, \quad(n \geq 0)
$$

and the Stirling numbers of the second kind are given by

$$
x^{n}=\sum_{l=0}^{n} S_{2}(n, l)(x)_{l},
$$

where $(x)_{0}=1$ and $(x)_{n}=x(x-1) \cdots(x-n+1)(n \geq 1)$ (see $[4,5])$.

From (4) and (5), we can derive the following equations:

$$
\left(e^{t}-1\right)^{n}=n ! \sum_{l=n}^{\infty} S_{2}(l, n) \frac{t^{l}}{l !},
$$

$$
(\log (x+1))^{n}=n ! \sum_{l=n}^{\infty} S_{1}(l, n) \frac{x^{l}}{l !}, \quad(n \geq 0),
$$

In addition,

$$
\text { (see }[4-6]) \text {. }
$$

$$
\log (1+t)=\sum_{n=1}^{\infty}(-1)^{n+1}(n-1) ! \frac{t^{n}}{n !}
$$


The Bernoulli polynomials of order $r$ are defined by the following generating function:

$$
\left(\frac{t}{e^{t}-1}\right)^{r} e^{x t}=\sum_{n=0}^{\infty} B_{n}^{(r)}(x) \frac{t^{n}}{n !}
$$

(see [7-9]).

Carlitz's degenerate Bernoulli polynomials of order $r$ is defined by the generating function to be

$$
\sum_{n=0}^{\infty} \beta_{n}^{(r)}(x \mid \lambda) \frac{t^{n}}{n !}=\left(\frac{t}{(1+\lambda t)^{1 / \lambda}-1}\right)^{r}(1+\lambda t)^{x / \lambda}
$$

where $\lambda \in \mathbb{R}$ (see [10]). By (10), we know that

$$
\begin{aligned}
\lim _{\lambda \longrightarrow 0} \sum_{n=0}^{\infty} \beta_{n}^{(r)}(x \mid \lambda) \frac{t^{n}}{n !} & =\lim _{\lambda \longrightarrow 0}\left(\frac{t}{(1+\lambda t)^{1 / \lambda}-1}\right)^{r}(1+\lambda t)^{x / \lambda} \\
& =\left(\frac{t}{e^{t}-1}\right)^{r} e^{x t}=\sum_{n=0}^{\infty} B_{n}^{(r)}(x) \frac{t^{n}}{n !}
\end{aligned}
$$

and thus, we obtain

$$
\lim _{\lambda \longrightarrow 0} \beta_{n}^{(r)}(x \mid \lambda)=B_{n}^{(r)}(x) .
$$

In [11], the degenerate Bernoulli polynomials are defined as

$$
\left(\frac{\log (1+\lambda t)^{1 / \lambda}}{(1+\lambda t)^{1 / \lambda}-1}\right)^{r}(1+\lambda t)^{x / \lambda}=\sum_{n=0}^{\infty} b_{n, \lambda}^{(r)}(x) \frac{t^{n}}{n !},
$$

which are different from Carlitz's degenerate numbers and polynomials.

By (13), we know that

$$
\lim _{\lambda \longrightarrow 0} b_{n, \lambda}(x)=B_{n}^{(r)}(x) .
$$

Note that by (3),

$$
\begin{aligned}
& \int_{\mathbb{Z}_{p}} \cdots \int_{\mathbb{Z}_{p}}(1+\lambda t)^{x+x_{1}+\cdots+x_{r} / \lambda} \mathrm{d} \mu_{0}\left(x_{1}\right) \ldots \mathrm{d} \mu_{0}\left(x_{r}\right) \\
& =\left(\frac{(1 / \lambda) \log (1+\lambda t)}{(1+\lambda t)^{1 / \lambda}-1}\right)^{r}(1+\lambda t)^{x / \lambda}=\sum_{n=0}^{\infty} b_{n, \lambda}^{(r)}(x) \frac{t^{n}}{n !}, \\
& \int_{\mathbb{Z}_{p}} \cdots \int_{\mathbb{Z}_{p}}(1+\lambda t)^{x+x_{1}+\cdots+x_{r} / \lambda} \mathrm{d} \mu_{0} \cdots \mathrm{d} \mu_{0}\left(x_{r}\right) \\
& =\int_{\mathbb{Z}_{p}} \cdots \int_{\mathbb{Z}_{p}} \sum_{n=0}^{\infty}\left(\frac{x+x_{1}+\cdots+x_{r}}{\lambda}\right) \lambda^{n} t^{n} \mathrm{~d} \mu_{0}\left(x_{1}\right) \cdots \mathrm{d} \mu_{0}\left(x_{r}\right) \\
& =\sum_{n=0}^{\infty} \int_{\mathbb{Z}_{p}} \cdots \int_{\mathbb{Z}_{p}}\left(x+x_{1}+\cdots+x_{r}\right)_{n, \lambda} \mathrm{d} \mu_{0}\left(x_{1}\right) \cdots \mathrm{d} \mu_{0}\left(x_{r}\right) \frac{t^{n}}{n !} .
\end{aligned}
$$

By (15) and (16), we know that

$b_{n, \lambda}^{(r)}(x)=\int_{\mathbb{Z}_{p}} \cdots \int_{\mathbb{Z}_{p}}\left(x+x_{1}+\cdots+x_{r}\right)_{n, \lambda} \mathrm{d} \mu_{0}\left(x_{1}\right) \cdots \mathrm{d} \mu_{0}\left(x_{r}\right)$.

The higher-order Daehee polynomials are defined by the generating function to be

$$
\left(\frac{\log (1+t)}{t}\right)^{r}(1+t)^{x}=\sum_{n=0}^{\infty} D_{n}^{(r)}(x) \frac{t^{n}}{n !}
$$

(see $[12-15])$.

In particular, if $r=1$, then $D_{n}^{(1)}(x)=D_{n}(x)$ is called the Daehee polynomials.

By replacing $t$ as $\log (1+t)$ in (18), we have

$$
\begin{aligned}
\sum_{n=0}^{\infty} D_{n}^{(r)}(x) \frac{t^{n}}{n !} & =\sum_{n=0}^{\infty} B_{n}^{(r)}(x) \frac{1}{n !}(\log (1+t))^{n} \\
& =\left(\sum_{n=0}^{\infty} B_{n}^{(r)}(x) \frac{1}{n !}\right)\left(n ! \sum_{l=0}^{\infty} S_{1}(l, n) \frac{t^{l}}{l !}\right) \\
& =\sum_{n=0}^{\infty}\left(\sum_{m=0}^{n} B_{n}^{(r)}(x) S_{1}(n, m)\right) \frac{t^{n}}{n !}
\end{aligned}
$$

and so, we obtain

$$
D_{n}^{(r)}(x)=\sum_{m=0}^{n} B_{m}^{(r)}(x) S_{1}(n, m) .
$$

Note that by (3), we have 


$$
\begin{aligned}
\sum_{n=0}^{\infty} D_{n}^{(r)}(x) \frac{t^{n}}{n !} & =\left(\frac{\log (1+t)}{t}\right)^{r}(1+t)^{x} \\
& =\int_{\mathbb{Z}_{p}} \cdots \int_{\mathbb{Z}_{p}}(1+t)^{x+x_{1}+\cdots+x_{r}} \mathrm{~d} \mu_{0}\left(x_{1}\right) \cdots \mathrm{d} \mu_{0}\left(x_{r}\right) \\
& =\int_{\mathbb{Z}_{p}} \cdots \int_{\mathbb{Z}_{p}}\left(\begin{array}{c}
x+x_{1}+\cdots+x_{r} \\
n
\end{array}\right) t^{n} \mathrm{~d} \mu_{0}\left(x_{1}\right) \cdots \mathrm{d} \mu_{0}\left(x_{r}\right) \\
& =\sum_{n=0}^{\infty}\left(\int_{\mathbb{Z}_{p}} \cdots \int_{\mathbb{Z}_{p}}\left(x+x_{1}+\cdots+x_{r}\right)_{n} \mathrm{~d} \mu_{0}\left(x_{1}\right) \cdots \mathrm{d} \mu_{0}\left(x_{r}\right)\right) \frac{t^{n}}{n !},
\end{aligned}
$$

and thus, we know that

$$
D_{n}^{(r)}(x)=\int_{\mathbb{Z}_{p}} \cdots \int_{\mathbb{Z}_{p}}\left(x+x_{1}+\cdots+x_{r}\right)_{n} \mathrm{~d} \mu_{0}\left(x_{1}\right) \cdots \mathrm{d} \mu_{0}\left(x_{r}\right) .
$$

Carlitz introduced the degenerate Bernoulli polynomials in [10] and the degeneration of special functions have been studied (see [6, 16-29]).

In particular, the degenerate Stirling numbers of the second kind with a generating function are defined as

$$
\frac{1}{m !}\left((1+\lambda t)^{1 / \lambda}-1\right)^{m}=\sum_{n=m}^{\infty} S_{2, \lambda}(n, m) \frac{t^{n}}{n !},
$$

where $m$ is a given nonnegative integer in $[6,10,22,30]$.

After introducing Daehee numbers and polynomials [31], it plays an important role of developing various generalized polynomials, and interesting properties are obtained (see $[8,15,21,22,28,30-35]$ ).

In this paper, we define the new degenerate Daehee polynomials and numbers which are called the degenerate Daehee polynomials of the second kind and investigate identities and properties of new polynomials.

\section{Fully Degenerate Daehee Polynomials of the Second Kind}

Let us assume that $\lambda \in \mathbb{R}$. By (3), we have

$$
\begin{aligned}
\int_{\mathbb{Z}_{p}} & (1+\lambda \log (1+t))^{x+y / \lambda} \mathrm{d} \mu_{0}(y) \\
= & \frac{(1 / \lambda) \log (1+\lambda \log (1+t))}{(1+\lambda \log (1+t))^{1 / \lambda}-1}(1+\lambda \log (1+t))^{x / \lambda} .
\end{aligned}
$$

By (24), we define the degenerate Daehee polynomials of the second kind by the generating function to be

$$
\begin{aligned}
& \frac{(1 / \lambda) \log (1+\lambda \log (1+t))}{(1+\lambda \log (1+t))^{1 / \lambda}-1}(1+\lambda \log (1+t))^{x / \lambda} \\
& =\sum_{n=0}^{\infty} D_{n}(x \mid \lambda) \frac{t^{n}}{n !}
\end{aligned}
$$

In the special case, $x=0$, and $D_{n}(\lambda)=D_{n}(0 \mid \lambda)$ are called the degenerate Daehee numbers of the second kind.

Note that

$$
\begin{aligned}
\lim _{\lambda \longrightarrow 0} \sum_{n=0}^{\infty} D_{n}(x \mid \lambda) \frac{t^{n}}{n !}= & \lim _{\lambda \longrightarrow 0} \frac{(1 / \lambda) \log (1+\lambda \log (1+t))}{(1+\lambda \log (1+t))^{1 / \lambda}-1} \\
& \cdot(1+\lambda \log (1+t))^{x / \lambda} \\
= & \frac{\log (1+t)}{t}(1+t)^{x}=\sum_{n=0}^{\infty} D_{n}(x) \frac{t^{n}}{n !}
\end{aligned}
$$

and thus, we know that

$$
\lim _{\lambda \rightarrow 0} D_{n}(x \mid \lambda)=D_{n}(x), \quad(n \geq 0) .
$$

From (7) and (24), we have

$$
\begin{aligned}
\int_{\mathbb{Z}_{p}} & (1+\lambda \log (1+t))^{x+y / \lambda} \mathrm{d} \mu_{0}(y) \\
= & \sum_{l=0}^{\infty} \int_{\mathbb{Z}_{p}}\left(\begin{array}{c}
\frac{x+y}{\lambda} \\
l
\end{array}\right) \lambda^{l}(\log (1+t))^{l} \mathrm{~d} \mu_{0}(y) \\
= & \sum_{n=0}^{\infty}\left(\sum_{k=0}^{n} S_{1}(n, l) \lambda^{l} l ! \int_{\mathbb{Z}_{p}}\left(\begin{array}{c}
\frac{x+y}{\lambda} \\
l
\end{array}\right) \mathrm{d} \mu_{0}(y)\right) \frac{t^{n}}{n !} .
\end{aligned}
$$

By (25) and (28), we have

$$
D_{n, \lambda}=\sum_{k=0}^{n} S_{1}(n, l) \lambda^{l} l ! \int_{\mathbb{Z}_{p}}\left(\begin{array}{c}
\frac{x+y}{\lambda} \\
l
\end{array}\right) \mathrm{d} \mu_{0}(y), \quad(n \geq 0) .
$$

Since 


$$
\begin{aligned}
\left(\begin{array}{c}
\frac{x+y}{\lambda} \\
l
\end{array}\right) & =\frac{((x+y) / \lambda)(((x+y) / \lambda)-1)(((x+y) / \lambda)-2) \ldots(((x+y) / \lambda)-l+1)}{l !} \\
& =\frac{(x+y)(x+y-\lambda)(x+y-2 \lambda) \ldots(x+y-(l-1) \lambda)}{\lambda^{l} l !}
\end{aligned}
$$

by (29) and (30), we have

$$
D_{n}(\lambda)=\sum_{k=0}^{n} S_{1}(n, l) \int_{\mathbb{Z}_{p}}(x+y)_{l, \lambda} \mathrm{d} \mu_{0}(y), \quad(n \geq 0),
$$

where $(x)_{n, \lambda}=x(x-\lambda)(x-2 \lambda) \cdots(x-(n-1) \lambda)$.

Thus, by (29) and (30), we have the following theorem which is Witt's type formula about degenerate Daehee polynomials of the second kind.

Theorem 1. For each $n \geq 0$, we have

$$
\begin{aligned}
D_{n}(\lambda) & =\sum_{k=0}^{n} S_{1}(n, l) \lambda^{l} l ! \int_{\mathbb{Z}_{p}}\left(\begin{array}{c}
\frac{x+y}{\lambda} \\
l
\end{array}\right) \mathrm{d} \mu_{0}(y) \\
& =\sum_{k=0}^{n} S_{1}(n, l) \int_{\mathbb{Z}_{p}}(x+y)_{l, \lambda} \mathrm{d} \mu_{0}(y) .
\end{aligned}
$$

By replacing $t$ as $e^{t}-1$ in (25), we obtain the following:

$$
\begin{aligned}
\sum_{n=0}^{\infty} D_{n}(x \mid \lambda) \frac{\left(e^{t}-1\right)^{n}}{n !} & =\sum_{n=0}^{\infty} D_{n}(x \mid \lambda) \sum_{m=n}^{\infty} S_{2}(m, n) \frac{t^{m}}{m !} \\
& =\sum_{n=0}^{\infty}\left(\sum_{m=0}^{n} D_{m}(x \mid \lambda) S_{2}(m, n)\right) \frac{t^{n}}{n !} .
\end{aligned}
$$

On the other hand,

$$
\begin{aligned}
\sum_{n=0}^{\infty} D_{n}(x \mid \lambda) \frac{\left(e^{t}-1\right)^{n}}{n !} & =\frac{(1 / \lambda) \log (1+\lambda t)}{(1+\lambda t)^{1 / \lambda}-1}(1+\lambda t)^{x / \lambda} \\
& =\sum_{n=0}^{\infty} B_{n}(x \mid \lambda) \frac{t^{n}}{n !}
\end{aligned}
$$

where $B_{n}(x \mid \lambda)$ is the degenerate Bernoulli polynomials of the second kind of order $r \in \mathbb{Z}$ which are defined by the generating function to be

$$
\left(\frac{(1 / \lambda) \log (1+\lambda t)}{(1+\lambda t)^{1 / \lambda}-1}\right)^{r}(1+\lambda t)^{x / \lambda}=\sum_{n=0}^{\infty} B_{n}^{(r)}(x \mid \lambda) \frac{t^{n}}{n !}
$$

(see $[20,25])$.

In particular, if $r=1, B_{n}^{(1)}(x \mid \lambda)=B_{n}(x \mid \lambda)$ is called the degenerate Bernoulli polynomials of the second kind.

For positive integer $d$ with $d \equiv 1(\bmod 2)$, if we put $f(x)=(1+\lambda \log (1+t))^{x / \lambda}$, then, by (2), we obtain

$$
\begin{aligned}
\int_{\mathbb{Z}_{p}} & (1+\lambda \log (1+t))^{x+d / \lambda} \mathrm{d} \mu_{0}(y) \\
& -\int_{\mathbb{Z}_{p}}(1+\lambda \log (1+t))^{x / \lambda} \mathrm{d} \mu_{0}(y) \\
= & \sum_{l=0}^{d-1} \frac{1}{\lambda}(1+\lambda \log (1+t))^{x / \lambda} \log (1+\lambda \log (1+t)) .
\end{aligned}
$$

By (36), we have

$$
\begin{aligned}
\int_{\mathbb{Z}_{p}} & (1+\lambda \log (1+t))^{x / \lambda} \mathrm{d} \mu_{0}(x) \\
= & \frac{(1 / \lambda) \log (1+\lambda \log (1+t))}{(1+\lambda \log (1+t))^{d / \lambda}-1} \sum_{l=0}^{d-1}(1+\lambda \log (1+t))^{l / \lambda} \\
= & d \sum_{l=0}^{d-1} \frac{(1 / \lambda) \log (1+(\lambda / d) d \log (1+t))}{(1+(\lambda / d) d \log (1+t))^{d / \lambda}-1} \\
& \left(1+\frac{\lambda}{d} d \log (1+t)\right)^{(d / \lambda)(l / d)} .
\end{aligned}
$$

Note that by (6),

$$
\begin{aligned}
& \frac{(1 / \lambda) \log (1+(\lambda / d) d \log (1+t))}{(1+(\lambda / d) d \log (1+t))^{(d / \lambda)}-1}(1+(\lambda / d) d \log (1+t))^{(d / \lambda)(l / d)} \\
& =\sum_{n=0}^{\infty} B_{n}\left(\frac{l}{d} \mid \frac{\lambda}{d}\right) \frac{(d \log (1+t))^{n}}{n !} \\
& =\sum_{n=0}^{\infty} B_{n}\left(\frac{l}{d} \mid \frac{\lambda}{d}\right) \frac{d^{n}}{n !} n ! \sum_{m=n}^{\infty} S_{1}(m, n) \frac{x^{m}}{m !} \\
& =\sum_{n=0}^{\infty}\left(\sum_{m=0}^{n} B_{m}\left(\frac{l}{d} \mid \frac{\lambda}{d}\right) d^{m} S_{1}(n, m)\right) \frac{t^{n}}{n !} .
\end{aligned}
$$

By (24), (37), and (38), we have

$$
\begin{aligned}
\sum_{n=0}^{\infty} D_{n}(\lambda) \frac{t^{n}}{n !} & =\int_{\mathbb{Z}_{p}}(1+\lambda \log (1+t))^{x / \lambda} \mathrm{d} \mu_{0}(x) \\
& =\sum_{n=0}^{\infty}\left(\sum_{m=0}^{n} \sum_{l=0}^{d-1} B_{m}\left(\frac{l}{d} \mid \frac{\lambda}{d}\right) d^{m+1} S_{1}(n, m)\right) \frac{t^{n}}{n !} .
\end{aligned}
$$


Hence, by (33), (34), and (39), we obtain the following theorem which shows the relationship between degenerate Daehee polynomials of the second kind and degenerate Bernoulli polynomials of the second kind.

Theorem 2. For nonnegative integer $n$ and $d \in \mathbb{N}$ with $d \equiv 1(\bmod 2)$, we have

$$
\begin{aligned}
B_{n}(x \mid \lambda)(x) & =\sum_{m=0}^{n} D_{m, \lambda}(x) S_{2}(n, m), \\
D_{n, \lambda} & =\sum_{m=0}^{n} \sum_{l=0}^{d-1} B_{m}\left(\frac{l}{d} \mid \frac{\lambda}{d}\right) d^{m+1} S_{1}(n, m) .
\end{aligned}
$$

By (25), we note that

$$
\begin{aligned}
\sum_{n=0}^{\infty} D_{n}(x \mid \lambda) \frac{t^{n}}{n !}= & \left(\frac{(1 / \lambda) \log (1+\lambda \log (1+t))}{(1+\lambda \log (1+t))^{1 / \lambda}-1}\right) \\
& \cdot(1+\lambda \log (1+t))^{x / \lambda} \\
= & \left(\sum_{n=0}^{\infty} D_{n}(\lambda) \frac{t^{n}}{n !}\right)\left(\sum_{n=0}^{\infty}\left(\begin{array}{c}
x \\
\lambda \\
n
\end{array}\right) \lambda^{n}(\log (1+t))^{n}\right) \\
= & \left(\sum_{n=0}^{\infty} D_{n}(\lambda) \frac{t^{n}}{n !}\right)\left(\sum_{n=0}^{\infty}\left(\begin{array}{c}
x \\
\lambda \\
n
\end{array}\right) \lambda^{n} n ! \sum_{l=n}^{\infty} S_{1}(l, n) \frac{x^{l}}{l !}\right) \\
= & \left(\sum_{n=0}^{\infty} D_{n}(\lambda) \frac{t^{n}}{n !}\right)\left(\sum_{n=0}^{\infty} \sum_{l=0}^{n}(x)_{l, \lambda} S_{1}(n, l) \frac{x^{n}}{n !}\right) \\
= & \sum_{n=0}^{\infty}\left(\sum_{k=0}^{n} \sum_{l=0}^{k}\left(\begin{array}{l}
n \\
k
\end{array}\right)(x)_{l, \lambda} S_{1}(k, l) D_{n-k}(\lambda)\right) \frac{t^{n}}{n !} .
\end{aligned}
$$

By comparing the coefficients on both sides of (41), we obtain the following theorem.

Theorem 3. For nonnegative integer $n$, we have

$$
D_{n}(x \mid \lambda)=\sum_{k=0}^{n} \sum_{l=0}^{k}\left(\begin{array}{l}
n \\
k
\end{array}\right)(x)_{l, \lambda} S_{1}(k, l) D_{n-k}(\lambda) .
$$

Note that if we put $f(x)=(1+\lambda \log (1+t))^{x / \lambda}$, then

$$
\begin{aligned}
f^{\prime}(0) & =\frac{1}{\lambda} \log (1+\lambda \log (1+t)) \\
& =\frac{1}{\lambda} \sum_{n=1}^{\infty}(-1)^{n+1} \frac{\lambda^{n}}{n}(\log (1+t))^{n} \\
& =\sum_{n=1}^{\infty}(-1)^{n+1} \frac{\lambda^{n-1}}{n} n ! \sum_{l=n}^{\infty} S_{1}(l, n) \frac{t^{l}}{l !} \\
& =\sum_{n=0}^{\infty}\left(\sum_{m=1}^{n}(-1)^{m+1} \lambda^{m-1}(m-1) ! S_{1}(n, m)\right) \frac{t^{n}}{n !},
\end{aligned}
$$

and thus, by (3), we have

$$
\begin{aligned}
\int_{\mathbb{Z}_{p}} f\left(x_{1}\right) \mathrm{d} \mu_{0}(x)-\int_{\mathbb{Z}_{p}} f(x) \mathrm{d} \mu_{0}(x) \\
=\frac{(1 / \lambda) \log (1+\lambda \log (1+t))}{(1+\lambda \log (1+t))^{1 / \lambda}-1}(1+\lambda \log (1+t))^{1 / \lambda} \\
-\frac{(1 / \lambda) \log (1+\lambda \log (1+t))}{(1+\lambda \log (1+t))^{1 / \lambda}-1} \\
=\sum_{n=0}^{\infty}\left(D_{n}(1 \mid \lambda)-D_{n}(\lambda)\right) \frac{t^{n}}{n !}
\end{aligned}
$$

Moreover,

$$
\begin{gathered}
((1 / \lambda) \log (1+\lambda \log (1+t))) \sum_{l=0}^{n-1}(1+\lambda \log (1+t))^{l / \lambda} \\
=\frac{(1 / \lambda) \log (1+\lambda \log (1+t))}{(1+\lambda \log (1+t))^{1 / \lambda}}(1+\lambda \log (1+t))^{n} \\
-\frac{(1 / \lambda) \log (1+\lambda \log (1+t))}{(1+\lambda \log (1+t))^{1 / \lambda}} \\
=\sum_{r=0}^{\infty}\left\{D_{r}(n \mid \lambda)-D_{r}(\lambda)\right\} \frac{t^{r}}{r !},
\end{gathered}
$$

and, by (43), we obtain 


$$
\begin{aligned}
& (1 / \lambda) \log (1+\lambda \log (1+t)) \sum_{l=0}^{n-1}(1+\lambda \log (1+t))^{l / \lambda} \\
& =\left(\sum_{p=0}^{\infty} \sum_{m=1}^{p}(-\lambda)^{m-1}(m-1) ! S-1(p, m) \frac{t^{p}}{p !}\right) \\
& \cdot\left(\sum_{l=0}^{n-1} \sum_{s=0}^{\infty} \sum_{q=0}^{s}(l)_{q, \lambda} S_{1}(s, q) \frac{t^{s}}{s !}\right) \\
& =\sum_{r=0}^{\infty}\left(\sum_{l=0}^{n-1} \sum_{p=0}^{r} \sum_{m=1}^{p} \sum_{q=0}^{r-p}\left(\begin{array}{c}
r \\
p
\end{array}\right)(l)_{q, \lambda}(-\lambda)^{m-1}\right. \\
& \left.\cdot(m-1) ! S_{1}(p, m) S_{1}(r-p, q)\right) \frac{t^{r}}{r !} .
\end{aligned}
$$

From (43), (44), and (46), we obtain the following theorem which represents a recurrence relations between degenerate Daehee polynomials of the second kind and degenerate Daehee numbers of the second kind.

Theorem 4. For each nonnegative integer $r$, we have

$$
D_{r}(1 \mid \lambda)-D_{r}(\lambda)=\sum_{m=1}^{r}(-\lambda)^{m-1}(m-1) ! S_{1}(r, m) .
$$

Moreover, for each positive integer $n \geq 2$,

$$
\begin{aligned}
D_{r}(n \mid \lambda)-D_{r}(\lambda)= & \sum_{l=0}^{n-1} \sum_{p=0}^{r} \sum_{m=1}^{p} \sum_{q=0}^{r-p}\left(\begin{array}{c}
r \\
p
\end{array}\right)(l)_{q, \lambda}(-\lambda)^{m-1} \\
& \cdot(m-1) ! S_{1}(p, m) S_{1}(r-p, q) .
\end{aligned}
$$

\section{Higher-Order Degenerate Daehee Polynomials of the Second Kind}

In this section, we consider the higher-order degenerate Daehee polynomials of the second kind given by the generating function as follows: for the given positive real number $r$,

$$
\begin{aligned}
& \left(\frac{(1 / \lambda) \log (1+\lambda \log (1+t))}{(1+\lambda \log (1+t))^{1 / \lambda}-1}\right)^{r}(1+\lambda \log (1+t))^{x / \lambda} \\
& \quad=\sum_{n=0}^{\infty} D_{n}^{(r)}(x \mid \lambda) \frac{t^{n}}{n !}
\end{aligned}
$$

In particular, if $x=0, D_{n}^{(r)}(0 \mid \lambda)=D_{n}^{(r)}(\lambda)$ are called the higher-order degenerate Daehee numbers of the second kind. Note that

$$
\begin{aligned}
\lim _{\lambda \longrightarrow 0} \sum_{n=0}^{\infty} D_{n}^{(r)}(x \mid \lambda) \frac{t^{n}}{n !}= & \lim _{\lambda \longrightarrow 0}\left(\frac{(1 / \lambda) \log (1+\lambda \log (1+t))}{(1+\lambda \log (1+t))^{1 / \lambda}-1}\right)^{r} \\
& \cdot(1+\lambda \log (1+t))^{x / \lambda} \\
= & \left(\frac{\log (1+t)}{t}\right)^{r}(1+t)^{x} \\
= & \sum_{n=0}^{\infty} D_{n}^{(r)}(x) \frac{t^{n}}{n !}
\end{aligned}
$$

From (35), we note that

$$
\begin{aligned}
& \left(\frac{(1 / \lambda) \log (1+\lambda \log (1+t))}{(1+\lambda \log (1+t))^{1 / \lambda}-1}\right)^{r}(1+\lambda \log (1+t))^{x / \lambda} \\
& =\sum_{n=0}^{\infty} B_{n}^{(r)}(x \mid \lambda) \frac{(\log (1+t))^{n}}{n !} \\
& =\sum_{n=0}^{\infty} \frac{B_{n}^{(r)}(x \mid \lambda)}{n !} n ! \sum_{l=n}^{\infty} S_{1}(l, n) \frac{t^{l}}{l !} \\
& =\sum_{n=0}^{\infty}\left(\sum_{m=0}^{n} B_{m}^{(r)}(x \mid \lambda) S_{1}(n, m)\right) \frac{t^{n}}{n !} .
\end{aligned}
$$

In addition, by replacing $t$ by $e^{t}-1$ in (49), we have

$$
\begin{aligned}
\sum_{n=0}^{\infty} D_{n}^{(r)}(x \mid \lambda) \frac{\left(e^{t}-1\right)^{n}}{n !} & =\sum_{n=0}^{\infty} \frac{D_{n}^{(r)}(x \mid \lambda)}{n !} n ! \sum_{m=n}^{\infty} S_{2}(n, m) \frac{t^{m}}{m !} \\
& =\sum_{n=0}^{\infty}\left(\sum_{m=0}^{n} D_{m}^{(r)}(x \mid \lambda) S_{2}(n, m)\right) \frac{t^{n}}{n !}
\end{aligned}
$$

$$
\begin{aligned}
\sum_{n=0}^{\infty} D_{n}^{(r)}(x \mid \lambda) \frac{\left(e^{t}-1\right)^{n}}{n !} & =\left(\frac{(1 / \lambda) \log (1+\lambda t)}{(1+\lambda t)^{1 / \lambda}-1}\right)^{r}(1+\lambda t)^{x / \lambda} \\
& =\sum_{n=0}^{\infty} B_{n}^{(r)}(x \mid \lambda) \frac{t^{n}}{n !}
\end{aligned}
$$

Hence, by (51)-(53), we obtain the following theorem.

Theorem 5. For $n \geq 0$, we have

$$
D_{n}^{(r)}(x \mid \lambda)=\sum_{m=0}^{n} B_{m}^{(r)}(x \mid \lambda) S_{1}(n, m) .
$$


Moreover,

$$
B_{n}^{(r)}(x \mid \lambda)=\sum_{m=0}^{n} D_{m}^{(r)}(x \mid \lambda) S_{2}(n, m) .
$$

In particular, if $r=1$, then we know that Theorem 5 is a generalization of Theorem 1 and Theorem 2 .

Note that for each $k, r \in \mathbb{N}$,

$$
\begin{aligned}
\left(\frac{(1 / \lambda) \log (1+\lambda \log (1+t))}{(1+\lambda \log (1+t))^{1 / \lambda}-1}\right)^{r}(1+\lambda \log (1+t))^{x / \lambda} \\
=\left(\frac{(1 / \lambda) \log (1+\lambda \log (1+t))}{(1+\lambda \log (1+t))^{1 / \lambda}-1}\right)^{k} \\
\\
\cdot\left(\frac{(1 / \lambda) \log (1+\lambda \log (1+t))}{(1+\lambda \log (1+t))^{1 / \lambda}-1}\right)^{r-k}(1+\lambda \log (1+t))^{x / \lambda} \\
=\left(\sum_{n=0}^{\infty} D_{n}^{(k)}(\lambda) \frac{t^{n}}{n !}\right)\left(\sum_{n=0}^{\infty} D_{n}^{(r-k)}(x \mid \lambda) \frac{t^{n}}{n !}\right) \\
=\sum_{n=0}^{\infty}\left(\sum_{m=0}^{n}\left(\begin{array}{c}
n \\
m
\end{array}\right) D_{m}^{(k)}(\lambda) D_{n-m}^{(r-k)}(x \mid \lambda)\right) \frac{t^{n}}{n !}
\end{aligned}
$$

It is well known that for each $k \in \mathbb{Z}$,

$$
\left(\frac{t}{\log (1+t)}\right)^{k}(1+t)^{x-1}=\sum_{n=0}^{\infty} B_{n}^{(n-k+1)}(x) \frac{t^{n}}{n !} .
$$

By (23), (7), and (57), we have

$$
\begin{aligned}
\left(\frac{(1 / \lambda) \log (1+\lambda \log (1+t))}{(1+\lambda \log (1+t))^{1 / \lambda}-1}\right)^{r}(1+\lambda \log (1+t))^{x / \lambda} \\
\quad=\left(\frac{(1 / \lambda) \log (1+\lambda \log (1+t))}{(1+\lambda \log (1+t))^{1 / \lambda}-1}\right)^{-r}(1+\lambda \log (1+t))^{x / \lambda} \\
=\sum_{n=0}^{\infty} B_{n}^{(n+r+1)}(x+1) \frac{1}{n !}\left((1+\lambda \log (1+t))^{1 / \lambda}-1\right)^{n} \\
=\left(\sum_{n=0}^{\infty} B_{n}^{(n+r+1)}(x+1)\right)\left(\sum_{m=n}^{\infty} S_{2, \lambda}(m, n) \frac{(\log (1+t))^{m}}{m !}\right) \\
=\sum_{p=0}^{\infty} \sum_{n=0}^{p} B_{n}^{(n+r+1)}(x+1) S_{2, \lambda}(p, n) \frac{1}{p !}(\log (1+t))^{p} \\
=\sum_{q=0}^{\infty}\left(\sum_{p=0}^{q} \sum_{n=0}^{p} B_{n}^{(n+r+1)}(x+1) S_{2, \lambda}(q-l, n) S_{1}(q, p)\right) \frac{t^{q}}{q !} .
\end{aligned}
$$

By (56) and (58), we obtain the following theorem.

$$
\begin{aligned}
& D_{n}^{(r)}(x \mid \lambda)=\sum_{m=0}^{n}\left(\begin{array}{c}
n \\
m
\end{array}\right) D_{m}^{(k)}(\lambda) D_{n-m}^{(r-k)}(x \mid \lambda), \\
& D_{q}^{(r)}(x \mid \lambda)=\sum_{p=0}^{q} \sum_{n=0}^{p} B_{n}^{(n+r+1)}(x+1) S_{2, \lambda}(q-l, n) S_{1}(q, p) .
\end{aligned}
$$

Note that by (3),

$$
\begin{aligned}
\int_{\mathbb{Z}_{p}} \cdots \int_{\mathbb{Z}_{p}}(1+\lambda \log (1+t))^{x+x_{1}+\cdots+x_{r} / \lambda} \mathrm{d} \mu_{0}\left(x_{1}\right) \cdots \mathrm{d} \mu_{0}\left(x_{r}\right) \\
=\left(\frac{(1 / \lambda) \log (1+\lambda \log (1+t))}{(1+\lambda \log (1+t))^{1 / \lambda}-1}\right)^{r}(1+\lambda \log (1+t))^{x / \lambda} \\
=\sum_{n=0}^{\infty} D_{n}^{(r)}(x \mid \lambda) \frac{t^{n}}{n !} .
\end{aligned}
$$

By (17) and (60), we have

$$
\begin{aligned}
\int_{\mathbb{Z}_{p}} & \cdots \int_{\mathbb{Z}_{p}}(1+\lambda \log (1+t))^{x+x_{1}+\cdots+x_{r} / \lambda} \mathrm{d} \mu_{0}\left(x_{1}\right) \cdots \mathrm{d} \mu_{0}\left(x_{r}\right) \\
= & \int_{\mathbb{Z}_{p}} \cdots \int_{\mathbb{Z}_{p}} \sum_{n=0}^{\infty}\left(\frac{x+x_{1}+\cdots+x_{r}}{\lambda}\right) \lambda^{n} \\
& \cdot(\log (1+t))^{n} \mathrm{~d} \mu\left(x_{1}\right) \cdots \mathrm{d} \mu_{0}\left(x_{r}\right) \\
= & \sum_{n=0}^{\infty} \int_{\mathbb{Z}_{p}} \cdots \int_{\mathbb{Z}_{p}}\left(x+x_{1}+\cdots+x_{r}\right)_{n, \lambda} \frac{1}{n !} n ! \sum_{l=n}^{\infty} S_{1}(l, n) \frac{x^{l}}{l !} \\
= & \sum_{n=0}^{\infty}\left(\sum_{m=0}^{n} \int_{\mathbb{Z}_{p}} \cdots \int_{\mathbb{Z}_{p}}\left(+x_{1}+\cdots+x_{r}\right)_{m, \lambda} S_{1}(n, m)\right) \frac{t^{n}}{n !} \\
= & \sum_{n=0}^{\infty}\left(\sum_{m=0}^{n} S_{1}(n, m) \beta_{m}^{(r)}(x)\right) \frac{t^{n}}{n !} .
\end{aligned}
$$

Thus, by (60) and (61), we obtain the following theorem which shows that higher-order degenerate Daehee polynomials of the second kind are represented by linear combination of the higher-order Carlitz's type degenerate Bernoulli polynomials.

Theorem 7. For each nonnegative integer $n$ and each integer $r$,

$$
D_{n}^{(r)}(x \mid \lambda)=\sum_{m=0}^{n} S_{1}(n, m) \beta_{m}^{(r)}(x) .
$$

By (7) and (23), we have

Theorem 6. For each $n, q \geq 0$, we have 


$$
\begin{aligned}
(1+ & \lambda \log (1+t))^{x+x_{1}+\cdots+x_{r} / \lambda} \\
= & \left((1+\lambda \log (1+t))^{1 / \lambda}-1+1\right)^{x+x_{1}+\cdots+x_{r}} \\
& =\sum_{m=0}^{\infty}\left(\begin{array}{c}
x+x_{1}+\cdots+x_{r} \\
m
\end{array}\right)\left((1+\lambda \log (1+t))^{1 / \lambda}-1\right)^{m} \\
& =\sum_{m=0}^{\infty}\left(x+x_{1}+\cdots+x_{r}\right)_{m} \sum_{n=m}^{\infty} S_{2, \lambda}(n, m) \frac{(\log (1+t))^{n}}{n !} \\
& =\sum_{n=0}^{\infty} \sum_{m=0}^{n}\left(x+x_{1}+\cdots+x_{r}\right)_{m} S_{2, \lambda}(n, m) \frac{(\log (1+t))^{n}}{n !} \\
& =\sum_{n=0}^{\infty} \sum_{m=0}^{n}\left(x+x_{1}+\cdots+x_{r}\right)_{m} S_{2, \lambda}(n, m) \frac{1}{n !} n ! \sum_{l=n}^{\infty} S_{1}(l, n) \frac{t^{l}}{l !} \\
& =\sum_{n=0}^{\infty}\left(\sum_{k=0}^{n} \sum_{m=0}^{k}\left(x+x_{1}+\cdots+x_{r}\right)_{m} S_{2, \lambda}(k, m) S_{1}(n, k)\right) \frac{t^{n}}{n !}
\end{aligned}
$$

and so by (22), (60), and (63), we obtain

$$
\begin{aligned}
\int_{\mathbb{Z}_{p}} \cdots \int_{\mathbb{Z}_{p}}(1+\lambda \log (1+t))^{x+x_{1}+\cdots+x_{r} / \lambda} \mathrm{d} \mu_{0}\left(x_{1}\right) \cdots \mathrm{d} \mu_{0}\left(x_{r}\right) \\
=\sum_{n=0}^{\infty}\left(\sum_{k=0}^{n} \sum_{m=0}^{k} S_{2, \lambda}(k, m) S_{1}(n, k) \int_{\mathbb{Z}_{p}}\right. \\
\left.\quad \ldots \int_{\mathbb{Z}_{p}}\left(x+x_{1}+\cdots+x_{r}\right)_{m} \mathrm{~d} \mu_{0}\left(x_{1}\right) \cdots \mathrm{d} \mu_{0}\left(x_{r}\right)\right) \frac{t^{n}}{n !} \\
=\sum_{n=0}^{\infty}\left(\sum_{k=0}^{n} \sum_{m=0}^{k} S_{2, \lambda}(k, m) S_{1}(n, k) D_{m}^{(r)}(x)\right) \frac{t^{n}}{n !} .
\end{aligned}
$$

By (60) and (64), we obtain the following theorem.

Theorem 8. For each nonnegative integer $m$,

$$
D_{n}^{(r)}(x \mid \lambda)=\sum_{k=0}^{n} \sum_{m=0}^{k} S_{2, \lambda}(k, m) S_{1}(n, k) D_{m}^{(r)}(x) .
$$

Theorem 8 shows that higher-order degenerate Daehee polynomials are related closely to Daehee polynomials of order $r$.

\section{Conclusion}

In the past two decades, the degenerations of special functions and their applications have been studied as a new area of mathematics. In this paper, we considered the degenerate Daehee numbers and polynomials by using $p$-adic invariant integral on $\mathbb{Z}_{p}$ which are different from Kim's degenerate Daehee polynomials. We derive some new and interesting properties of those polynomials.

Next, from the definition of the higher-order degenerate Daehee numbers and Daehee polynomials of the second kind, we found the relationship between the degenerate Bernoulli polynomials, the first and second Stirling numbers, the Bernoulli polynomials, degenerate Stirling numbers of the second kind, and those numbers and polynomials.

\section{Data Availability}

No data were used to support this study.

\section{Conflicts of Interest}

The authors declare that they have no conflicts of interest.

\section{Authors' Contributions}

All authors contributed equally to this work. All authors read and approved the final manuscript.

\section{Acknowledgments}

This research was supported by the Daegu University Research Grant, 2019.

\section{References}

[1] D. S. Kim and T. Kim, "Some $p$-adic integrals on $\mathbb{Z} p$ associated with trigonometric functions," Russian Journal of Mathematical Physics, vol. 25, no. 3, pp. 300-308, 2018.

[2] T. Kim, "Symmetryp-adic invariant integral on $\mathbb{Z} p$ for Bernoulli and Euler polynomials," Journal of Difference Equations and Applications, vol. 14, no. 12, pp. 1267-1277, 2008.

[3] T. Kim, "Multiple $p$-adic L-function," Russian Journal of Mathematical Physics, vol. 13, no. 2, pp. 151-157, 2006.

[4] L. Comtet, Advanced Combinatorics, Reidel, Dordrecht, Netherlands, 1974.

[5] S. Roman, The Umbral Calculus, Dover, New York, Ny, USA, 2005.

[6] D. S. Kim, T. Kim, and G. W. Jang, "A note on degenerate Stirling numbers of the first kind," Proceedings of the Jangjeon Mathematical Society, vol. 21, no. 3, pp. 394-404, 2018.

[7] A. Bayad and T. Kim, "Identities for the Bernoulli, the euler and the genocchi numbers and polynomials," Advanced Studies in Contemporary Mathematics (Kyungshang), vol. 20, no. 2, pp. 247-253, 2010.

[8] B. S. El-Desouky and A. Mustafa, "New results and matrix representation for daehee and Bernoulli numbers and polynomials," Applied Mathematical Sciences, vol. 9, pp. 35933610, 2015.

[9] Y. Simsek, "Generating functions of the twisted Bernoulli numbers and polynomials associated with their interpolation functions," Advanced Studies in Contemporary Mathematics (Kyungshang), vol. 16, no. 2, pp. 251-278, 2008.

[10] L. Carlitz, "Degenerate stirling, Bernoulli and eulerian numbers," Utilitas Mathematica, vol. 15, pp. 51-88, 1979.

[11] T. Kim, D. Kim, and H.-I. Kwon, "Some identities relating to degenerate Bernoulli polynomials," Filomat, vol. 30, no. 4, pp. 905-912, 2016. 
[12] S. Araci, M. Acikgoz, and U. Duran, "On weighted $q$-Daehee polynomials with their applications," Indagationes Mathematicae, vol. 30, no. 2, pp. 365-374, 2019.

[13] U. Duran and M. Acikgoz, "Multifarious correlations for $p$-adic gamma function and weighted $q$-Daehee polynomials," Maejo International Journal of Science and Technology, vol. 13, no. 3, pp. 209-216, 2019.

[14] W. A. Khan, K. S. Nisar, U. Duran, M. Acikgoz, and S. Araci, "Multifarious implicit summation formulae of Hermite-based poly-Daehee polynomials," Applied Mathematics \& Information Sciences, vol. 12, no. 2, pp. 305-310, 2018.

[15] D. S. Kim, T. Kim, S. H. Lee, and J. J. Seo, "Higher-order Daehee numbers and polynomials," International Journal of Mathematical Analysis, vol. 24, no. 1, pp. 5-18, 2014.

[16] J. Jeong and S.-H. Rim, "On finite times degenerate higherorder Cauchy numbers and polynomials," Bulletin of the Korean Mathematical Society, vol. 53, no. 5, pp. 1427-1437, 2016.

[17] W. A. Khan, "A note on degenerate Hermite poly-Bernoulli numbers and polynomials," Journal of Classical Analysis, vol. 8, no. 1, pp. 65-76, 2016.

[18] W. A. Khan and M. Ahmad, "Partially degenerate polyBernoulli polynomials associated with Hermite polynomials," Advanced Studies in Contemporary Mathematics (Kyungshang), vol. 28, no. 3, pp. 487-496, 2018.

[19] D. S. Kim and T. Kim, "A note on degenerate Eulerian numbers and polynomials," Advanced Studies in Contemporary Mathematics (Kyungshang), vol. 27, no. 4, pp. 431-440, 2017.

[20] D. S. Kim, T. Kim, and D. V. Dolgy, "A note on degenerate Bernoulli numbers and polynomials associated with $p$-adic invariant integral on $\mathbb{Z}_{p}$," Applied Mathematics and Computation, vol. 259, pp. 198-204, 2015.

[21] D. S. Kim, T. Kim, H. I. Kwon, and G. W. Jang, "Degenerate Daehee polynomials of the second kind," Proceedings of the Jangjeon Mathematical Society, vol. 21, no. 1, pp. 83-97, 2018.

[22] T. Kim, "A note on degenerate Strirling polynomials of the second kind," Proceedings of the Jangjeon Mathematical Society, vol. 20, no. 3, pp. 319-331, 2017.

[23] T. Kim and D. S. Kim, "Degenerate Laplace transform and degenerate gamma function," Russian Journal of Mathematical Physics, vol. 24, no. 2, pp. 241-248, 2017.

[24] T. Kim and D. S. Kim, "Identities for degenerate Bernoulli polynomials and Korobov polynomials of the first kind," Science China Mathematics, vol. 62, no. 5, pp. 999-1028, 2019.

[25] T. Kim and J. J. Seo, "Degenerate Bernoulli numbers and polynomials of the second kind," International Journal of Mathematical Analysis, vol. 9, no. 26, pp. 1269-1278, 2015.

[26] T. Kim, Y. Yao, D. S. Kim, and G.-W. Jang, "Degenerate r-stirling numbers and r-bell polynomials," Russian Journal of Mathematical Physics, vol. 25, no. 1, pp. 44-58, 2018.

[27] J. G. Lee and J. Kwon, "The modified degenerate $q$-Bernoulli polynomials arising from $p$-adic invariant integral on $\mathbb{Z}_{p}$." Advances in Difference Equations, vol. 2017, no. 1, 2017.

[28] J.-W. Park, B. M. Kim, and J. Kwon, "On a modified degenerate Daehee polynomials and numbers," The Journal of Nonlinear Sciences and Applications, vol. 10, no. 3, pp. 1108-1115, 2017.

[29] S. S. Pyo, "Degenerate Cauchy numbers and polynomials of the fourth kind," Advanced Studies in Contemporary Mathematics (Kyungshang), vol. 28, no. 1, pp. 127-138, 2018.

[30] T. Kim, D. S. Kim, and G. W. Jang, "Extended Stirling polynomials of the second kind and and extended Bell polynomials," Proceedings of the Jangjeon Mathematical Society, vol. 20, no. 3, pp. 365-376, 2017.

[31] D. S. Kim and T. Kim, "Daehee numbers and polynomials," Applied Mathematical Sciences, vol. 7, pp. 5969-5976, 2013.

[32] B. S. El-Desouky, A. Mustafa, and F. M. Abdel-Moneim, "Multiparameter higher order Daehee and Bernoulli numbers and polynomials," Applied Mathematics, vol. 8, pp. 775-785, 2017.

[33] D. S. Kim, T. Kim, H. I. Kwon, and J.-J. Seo, "Daehee polynomials with $q$-parameter," Advanced Studies in Theoretical Physics, vol. 8, no. 13, pp. 561-569, 2014.

[34] Y. Simsek, "Dentities on the Changhee numbers and Apostoltype Daehee polynomials," Advanced Studies in Contemporary Mathematics (Kyungshang), vol. 27, no. 2, pp. 199-212, 2017.

[35] Y. Simsek and A. Yardimci, "Applications on the ApostolDaehee numbers and polynomials associated with special numbers, polynomials and p-dic integrals," Advances in Difference Equations, vol. 2016, no. 1, 2016. 